\title{
Opening the door: creating a questionnaire to facilitate communication between parents and physicians when infants and young children have feeding disorders
}

Opening the Door: Creating a questionnaire to facilitate communication between parents and physicians when infants and young children have feeding challenges" has been accepted for publication in the Clinical Practice (therapy) considering the statements provided in the article as personal opinion of the author which was found not having any conflict or biasness towards anything. As the article was a perspective one, information provided by the author was considered as an opinion to be expressed through publication.

Publisher took decision to make the article online solely based on the reviewers suggestion which considered the article not but a personal opinion of the author. However, it is found that the author have some personal concerns and issues, therefore, being retracted from the journal .

Jaclyn Pederson $\mathrm{MHI}^{*}$

Strategic Initiatives, Feeding Matters,US

*Author for correspondence:

jpederson@feedingmatters.org 